OPEN ACCESS

Edited by:

Monica Fedele,

Istituto per l'Endocrinologia e

l'oncologia "Gaetano Salvatore"

(CNR), Italy

Reviewed by:

Paola Ungaro,

National Research Council (CNR), Italy

Guidalberto Manfioletti,

University of Trieste, Italy

*Correspondence:

Carlos Pérez-Plasencia

carlos.pplas@gmail.com

${ }^{t}$ These authors have contributed

equally to this work

Specialty section:

This article was submitted to

Molecular and Cellular Oncology,

a section of the journal

Frontiers in Oncology

Received: 05 March 2021 Accepted: 08 September 2021

Published: 08 October 2021

Citation:

Coronel-Hernández J,

Pérez-Yépez EA, Delgado-Waldo I,

Contreras-Romero C,

Jacobo-Herrera N, Cantú-De León D

and Pérez-Plasencia C (2021)

Aberrant Metabolism as Inductor of

Epigenetic Changes in Breast Cancer:

Therapeutic Opportunities.

Front. Oncol. 11:676562.

doi: 10.3389/fonc.2021.676562

\section{Aberrant Metabolism as Inductor of Epigenetic Changes in Breast Cancer: Therapeutic Opportunities}

\author{
Jossimar Coronel-Hernández ${ }^{1 \dagger}$, Eloy Andrés Pérez-Yépez ${ }^{1,2+}$, Izamary Delgado-Waldo ${ }^{1}$, \\ Carlos Contreras-Romero ${ }^{1}$, Nadia Jacobo-Herrera ${ }^{3}$, David Cantú-De León ${ }^{4}$ \\ and Carlos Pérez-Plasencia ${ }^{1,5 *}$ \\ 1 Laboratorio de Genómica, Instituto Nacional de Cancerología, Mexico City, Mexico, ${ }^{2}$ Cátedra-CONACYT, Dirección de \\ Cátedras, Consejo Nacional de Ciencia y Tecnología (CONACYT), Mexico City, Mexico, ${ }^{3}$ Unidad de Bioquímica, Instituto \\ Nacional de Ciencias Médicas y Nutrición, Salvador Zubirán, Mexico City, Mexico, 4 Unidad de Investigación en Cáncer, \\ Instituto Nacional de Cancerología, Mexico City, Mexico, ${ }^{5}$ Laboratorio de Genómica Funcional, Unidad de Biomedicina, \\ Facultad de Estudios Superiores Iztacala, Universidad Nacional Autónoma de México, Mexico City, Mexico
}

Aberrant metabolism is arising interest in the scientific community not only because of the role it plays in the development and establishment of the tumor mass but also the possibility of drug poisoning of key enzymes overexpressed in tumor cells. Moreover, tumor metabolism provides key molecules to maintain the epigenetic changes that are also an undisputed characteristic of each tumor type. This metabolic change includes the Warburg effect and alterations in key pathways involved in glutaminolysis, pentose phosphate, and unsaturated fatty acid biosynthesis. Modifications in all these pathways have consequences that impact genetics and epigenetics processes such as DNA methylation patterns, histone post-translational modifications, triggering oncogenes activation, and loss in tumor suppressor gene expression to lead the tumor establishment. In this review, we describe the metabolic rearrangement and its association with epigenetic regulation in breast cancer, as well as its implication in biological processes involved in cancer progression. A better understanding of these processes could help to find new targets for the diagnosis, prognosis, and treatment of this human health problem.

Keywords: breast cancer, metabolism, therapeutic targets, epigenetic modifications, glycolysis

\section{INTRODUCTION}

The progressive process of carcinogenesis induces molecular changes in the cell that enable it to survive in the environment, allowing it to proliferate and grow in unfavorable conditions. Within these changes is the so-called aberrant metabolism. Under standard conditions, normal cells depend on glycolysis to obtain energy; a pathway that triggers the cleavage of glucose to pyruvate. Pyruvate is processed into acetyl-CoA, which is catabolized through a cycle of tricarboxylic acids (TCA) to 
obtain energy in the form of ATP during oxidative phosphorylation (OXPHOS) (1). On the other hand, tumor cells reprogram the metabolism to satisfy the concentration of essential nutrients and energy, an action known as the Warburg effect (2). This effect consists of a change in obtaining ATP through the degradation of glucose into lactate in the presence of oxygen through enzyme lactate dehydrogenase (LDHA) $(3,4)$, which enhances ATP production, allowing biosynthesis of biomass to growth and the production of intermediates that promote proliferation and survival (5).

Recent research showed that the aberrant metabolism in cancer is not only involved in maintaining a high proliferative rate or survival but also have consequences that impact epigenetic mechanisms such as DNA methylation, histone post-translational modifications, chromatin remodeler functions, trigger oncogenes activation, and loss in tumor suppressor genes expression to lead the tumor establishment (6). The purpose of this review is to highlight the regulatory implication of the aberrant metabolism in breast cancer over the epigenetic landscape.

\section{METABOLIC PATHWAYS: THE MASTER REGULATORS OF THE GENE EXPRESSION IN BREAST CANCER}

\section{Glycolysis and TCA}

Glucose is the most abundant catabolite in blood and is the principal primary energy source of cancer cells. Normal cells take glucose from blood vessels and enter the cell through glucose transporter proteins (GLUT). Once in the cytoplasm, it is metabolized into two pyruvate molecules by the glycolysis pathway (7). In breast cancer, the glucose uptake is increased due to the overexpression and translocation of GLUT1 (8) to the cell membrane, enhancing glucose delivery into the cell. This glucose is employed directly for lactate production due to overactivation of AMPK (AMP-activated protein kinase) (9) and overexpression of 6-phosphofructose 2-kinase (6PF2K) (10). In breast cancer cells, pyruvate is usually processed into lactate, which is actively transported to the extracellular matrix due to the upregulation of monocarboxylate 1 (MCT1), an essential lactate transporter, giving them a glycolytic profile. Lactate plays a principal role in regulating gene transcription by inhibiting the HDAC (histone deacetylase) enzymes, promoting hyperacetylation in nucleosomes and active transcriptional state (11). Some reports mention that the histone $\mathrm{H} 4$ acetylation levels increase when cells are treated with lactate, promoting changes in gene expression that favors the cancer establishment (12). In breast cancer-associated fibroblasts, the overproduction of lactate induces tumor growth by demethylation of HIF- $1 \alpha$ in patients' tissue (13).

As a result of glycolysis and the metabolic shift orchestrated by the Warburg effect, the pyruvate that is not oxidized into lactate is dehydrogenated by pyruvate dehydrogenase complex (PDC), and it is turned into acetyl-CoA, which enters directly into the tricarboxylic acid cycle (TCA). This cycle, composed of a series of biochemical reactions, has a central role in energy production, macromolecule synthesis, and redox balance (14). In several types of cancer including breast, enzymes that participate in TCA such as isocitrate dehydrogenase (IDH), succinate dehydrogenase $(\mathrm{SDH})$, and fumarate hydratase $(\mathrm{FH})$ are deregulated, affecting enzymes involved in epigenetic processes (15).

Acetyl-CoA is the sole donor of the acetyl group for the acetylation mechanism not only of histones but in general in protein acetylation. It is a central molecule in metabolism as it participates in catabolic (glycolysis and beta-oxidation) and anabolic reactions (lipogenesis, steroid synthesis, acetylcholine synthesis, etc.) (16). HATs (Histone acetyltransferase) transfer the acetyl group from acetyl-CoA to the amino group of lysine in their target proteins to open the chromatin structure. On the other hand, HDACs (Histone desacetyltransferase) catalyze the opposite reaction; the HDACs remove the acetyl group by hydrolysis, modulating the transcriptional repression by closing the chromatin structure (17). Acetylation status could be used as a biomarker to differentiate between breast cancer subtypes. It has been observed, a genomic gain of acetylation of $\mathrm{H} 3 \mathrm{~K} 4$ in early stages of breast cancer cells, specifically, in genes associated with estrogen response and epithelial-mesenchymal transition (18). The mTOR complex, the principal regulator of cell growth in cancer, also stimulates acetyl-CoA synthesis through ATP citrate lyase hyperphosphorylation (19). Besides, overexpression or copy-number gain of acetyl-CoA synthetase 2 (ACS2) enhances acetyl-CoA production, correlating with breast cancer progression. Moreover, the Warburg effect promoted lipid biosynthesis using acetyl-CoA through acetyl-CoA carboxylase (ACACA) in the MCF7 breast cancer cell line (20). In MCF7, LCCP, and MCF75C cell lines, there was reported a nuclear overactivation of two acetyl-CoA-producing enzymes: PDC and ATP citrate lyase (ACYL); and their repression have a crucial impact on histone acetylation status, proliferation, and endocrine therapy resistance $(21,22)$.

Another key intermediate is the $\alpha$-ketoglutarate, which is required as co-substrate for JHDMs (Jumonji C domaincontaining histone demethylases) and TET (Ten-eleven translocation) proteins, which participate in histone and DNA demethylation. Also, JHDMs proteins, as JMJD5, interact directly with pyruvate kinase muscle isozymes (PKM) to change the metabolic flux; its inhibition decreases glucose metabolism (23). JMJD4 is considered essential for breast cancer progression given its role in chromosome segregation, enhancing mitotic segregation errors, and triggering cell proliferation (24). All these proteins are overactivated in breast cancer due to $\alpha$-ketoglutarate being overproduced by glycolysis or glutamine pathway.

\section{Glutamine Metabolism as a Driver of Epigenetic Changes}

Glutamine is a key amino acid that relieves the high growth rates in cancer cells. High amounts of glutamine are utilized for survival and proliferation. This amino acid is required not only for biosynthetic pathways (i.e., nucleic acid synthesis) but also for 
glutaminolysis, which is converted into TCA cycle intermediates and lactate (25). Thought this way, cancer cells get an extra energy source. The expression of enzymes involved in glutamine metabolism varies widely depending on the cancer type and is affected by tissue of origin and oncogenotypes. The glutamine addiction is suggested to be due to alterations in components of the glutaminolysis pathway in cancer cells. For example, the glutamine uptake principally through solute carrier family 1 neutral amino acid transported member 5 (SLC1A5), also known as ASCT2, is hyperactivated in several types of cancer, leading to the progression and malignancy $(26,27)$. In head and neck squamous cell carcinoma and breast cancer cells, the inhibition of glutamine transport abolishes cell growth and proliferation and increases apoptosis and autophagy (28). When glutamine is in the cell, it is converted into glutamate by the glutaminase enzyme (GLS). It has been demonstrated that GLS levels correlate with tumor growth rates and malignancy as well as poor prognosis (29). GLS is overexpressed in breast cancer, liver cancer, colorectal cancer, brain cancer, cervical cancer, lung cancer, prostate cancer, and melanoma (30-34). The important role of GLS overexpression in tumor progression is reflected in breast cancer which is related to high-grade tumors and high metastasis rates (35). Also, basal-like triple-negative and HER2+ breast tumors express large amounts of GLS, whereas luminal B tumors have elevated levels than luminal B tumors (36), and the deregulation of glutaminolysis by GLS knockdown induces breast tumor growth inhibition (37). Moreover, the decrease of two alternative transcripts of GLS (KGA and GAC) by alkyl benzoquinones and specific siRNA induces autophagy through mTORC1 inhibition (38). Additionally, it has been widely observed the role of glutamine metabolism in other cellular processes such as purine, pyrimidine, and non-essential amino acid synthesis, fatty acid synthesis, and the support of the effect of reactive oxygen species (ROS) to prevent apoptosis under stress-energy conditions during cancer progression (39). Furthermore, glutaminolysis components regulate signaling pathways that promote tumor growth in breast cancer. The mTOR signaling pathway is activated by glutamate dehydrogenase (GLUD) levels (40), and $\alpha$-ketoglutarate induces mTOR dimerization and activation (25, 37 ) to hyperactivated cell proliferation. Also, glutamine fasting induces low levels of STAT3 phosphorylation in high invasive cancer cells (41). For this reason, glutaminolysis inhibition by GLS or GLUD knocking down inhibits migration and invasion and the epithelial-mesenchymal transition (EMT) mediated by STAT3 (42). Alterations in components downstream of the glutaminolysis pathway also induces epigenetic changes that could lead to the repression of anti-oncogenes and trigger cancer progression. It has been demonstrated that mutations of the isocitrate dehydrogenase 1 and 2 induces the conversion of $\alpha$-ketoglutarate $(\alpha-K G)$ to 2 -hydroxyglutarate to inhibit DNA demethylases and histone demethylases, leading to DNA methylation and histone 3 methylation in lysine $(\mathrm{K})$ residues 9 , 27 , and $20(43,44)$. Also, glutaminolysis regulates histone demethylases as Jumonji domain-containing protein 3 (JMJD3) and ubiquitously transcribed tetratricopeptide repeat $\mathrm{X}$ chromosome (UTX) that are specific demethylase of lysine 27 of histone 3 (H3K27). Recently, Bai et al. (45) showed that in absence of glutamine, JMJD3 activity decreases, whereas $\mathrm{H} 3 \mathrm{~K} 27 \mathrm{me} 3$ levels are increased. It was also demonstrated that JMJD3 interacts with promoter regions of XIAP and survivin, in a glutamine-dependent manner promoting apoptosis resistance in idiopathic pulmonary fibrosis fibroblast. Moreover, the chemical inhibition of GLS induces the diminish of the H3K4me3 mark and increases the acetylation of lysine 16 of histone 4 (H4K16ac) to alter the expression of anti-apoptotic as well as metastatic-associated genes in human breast cancer cells $(46,47)$. Histone acetylation is another epigenetic mechanism that controls gene expression and regulates cancer development and progression. Due to the significant role of glutaminolysis in cancer biology, the use of several pathway components as therapeutic targets has been proposed (48). However, a glutaminolysis-focused therapy is not available for the clinical management of cancer patients. Therefore, studies that allow us a better understanding of the complexity of glutamine metabolism and its molecular effects in cancer are still needed.

\section{Lipid Biosynthesis, Lipolysis, and Derived Metabolites}

There is an astounding amount of information on the role of hyperactive lipogenesis in the maintenance of tumor progression. The role of lipid membranes in sustaining high rates of cell replication in the tumor mass is evident; however, other functions of key enzymes in lipid biosynthesis have been characterized. Fatty acid synthase (FASN) catalyzes palmitate biosynthesis using acetyl$\mathrm{CoA}$ and malonyl-CoA in the presence of NADPH; FASN is overexpressed in treatment-resistant mammary tumors $(49,50)$, and fatty acid syntheses are increased in brain metastases in mammary tumors (51). Other functions of FASN in addition to lipid synthesis are mainly associated with oncogenic signaling derived from tyrosine receptor kinases; it has been described that FASN can be directly phosphorylated by HER2, leading to the increased enzymatic activity of FASN enhancing tumor cell invasion and migration (52). One of the most important metabolites in lipid biosynthesis is Acetyl-CoA which is synthesized in the mitochondria via various reactions such as oxidative decarboxylation of pyruvate, catabolism of different amino acids, or beta-oxidation of fatty acids, among others. However, since lipid biosynthesis occurs in the cytosol, the generation of Acetyl-CoA is derived mainly from citrate synthesized in the mitochondria and transported to the cytosol where ACL (ATP-Citrate lyase) catalyzes its conversion to Acetyl-CoA (53). In turn, the metabolic pathway responsible for the oxidative degradation of fatty acids is b-oxidation, which provides ATP, NADPH, and acetyl-CoA, and is used in the acetylation of proteins.

Tumor characterizes for a deregulated chromatin architecture. In particular, the cancer stem cells (CSCs) have an open chromatin, where the main donor of acetyl groups to histones is the acetyl-CoA. For instance, histone $\mathrm{H} 4$ (H4K8ac, H4K12ac, and H4K16ac) acetylation plays a main role for the maintenance of the stem phenotype of TNBC cells cultured under hypoxic conditions (54). Besides, CSCs have a widely demonstrated participation in drug 
resistance. Hence the understanding of the metabolic pathways of this type of cells will bring light to different aspects of tumors and their treatment response.

\section{One Carbon Metabolism and Methylation}

Cytosine methylation is the epigenetic modification process most studied since the 1970s. Its mechanism consists of the addition of a methyl group at the 5 -position of the cytosine ring catalyzed by DNA methyltransferases. The importance of DNA methylation is that at the promoter level (methylation of CpG islands), hypermethylation promotes silencing of gene expression; whereas global methylation (associated with regions without $\mathrm{CpG}$ islands) maintains genomic stability $(55,56)$. The methylation mechanism occurs not only in DNA but also in RNA and proteins. Nonetheless, the epigenetic role is associated with the methylation of proteins involved in chromatin organization, particularly histones H3 and H4 (57). S-adenosylmethionine (SAM) is the methyl group donor in cellular metabolism; in general, methyl group transfer is catalyzed by methyltransferases (in the case of DNA-by-DNA methyltransferases, DNMTs), which oxidize SAM to S-adenosylhomocysteine. SAM is the product of the metabolism of one carbon that couples two different cycles, the folate cycle, and the methionine cycle (58). In breast cancer, all the isoforms of DNMT1, 3A, and 3B are overexpressed (59), and overexpression of DNMT3A was associated with poor prognosis in sporadic breast cancer (60). Cancer progression involves chromatin reorganization, a highly complex process in which regions near the promoters of tumor suppressor genes are hypermethylated, inhibiting their transcription; for example, the levels of BRCA-1 and MGMT hypermethylation may not have prognostic value in overall survival (61).

Chromatin organization is also regulated by histone methylation, which occurs at lysine and arginine residues in the tails of histones $\mathrm{H} 3$ and $\mathrm{H} 4$ (62). In humans, this reaction is catalyzed by histone methyl transferases (HMTs) (63). In breast cancer, an increase in SAM leads to the overactivation of HMTs, allowing the progression of the tumor phenotype (64). The methyltransferase Suv39h1 promotes epithelial-mesenchymal transition by adding the $\mathrm{H} 3 \mathrm{~K} 9 \mathrm{me} 3$ mark on the E-cadherin promoter (65). Also, Suv39h1 interacts with DNMT1 to hypermethylate the estrogen receptor-alpha (ER) promoter, silencing its expression (66). Therefore, the patterns of both DNA and histone methylation, mediated by aberrant metabolism in breast cancer, are highly relevant to tumor progression.

\section{THERAPEUTIC TARGETS}

The reprogramming of cellular metabolism is of high relevance in the hallmarks of cancer (67). Table 1 compiles information on different drugs used to block fundamental enzymes of tumor metabolism. Several reports have shown that alterations in glycolysis, glutamine, lipid, and folate metabolism in breast cancer cells could be used as therapeutic targets. Inhibitors of glycolytic enzymes and transporters of glycolytic products such as GLUT1, hexokinase $(\mathrm{HK})$, 6-phosphofructo 2-kinase-fructose-2, 6-biphosphatase E (PFKFB3), PMK2, LDHA, and monocarboxylate transporter 1 (MCT1) have been studied in numerous preclinical studies (7). The non-metabolizable glucose analog 2-deoxy-D-Glucose (2-DG) blocks the first step in glycolysis. It is phosphorylated by hexokinase to produce 2-DG-6P, which cannot be metabolized, reducing proliferation (78). It has demonstrated that 2-DG exhibits a cytotoxic effect in breast cancer cells with mitochondrial respiratory (79). Another report revealed that 2-DG acts as a radiation and drug sensitizer of breast cancer cells (80). Moreover, a current study using a murine model showed that the combination of 2-DG with oncolytic virotherapy (NDV) induced tumor cell death and inhibited tumor growth (78). At this moment, the effects of this inhibitor in normal and tumor cells of patients remain unexplored; thus, exhaustive clinical studies are desirable.

Glutaminolysis is also considered a potential target in cancer. A critical step in the utilization of glutamine is its conversion to glutamate by the mitochondrial enzyme glutaminase (81). Glutamine analogs as 6-diazo-5-oxo-L-norleucine, azaserine, and acivicin bind irreversibly to the active site of glutaminase, showing antitumoral activity (82). The molecule CB-839, a GLS inhibitor, was tested in cell lines derived from breast cancer tumors showing activity only in triple-negative subtype and not in Her2+ cells (83). But the low potency, poor metabolic stability, and low solubility of these drugs limit their potential for clinical development.

However, in vitro and in vivo studies have demonstrated that enzymes of lipid metabolism are involved in tumor development and progression, supporting the search for inhibitors to lipids metabolism components. For instance, inhibitors to Fatty Acid Synthase (FAS) as C75, orlistat, C93 have shown effects in stopping tumor growth of xenograft models $(77,84,85)$. Nevertheless, these inhibitors have limitations such as low cell

TABLE 1 | Drugs used to target tumor metabolism.

\begin{tabular}{|c|c|c|c|c|}
\hline Pathway & Inhibitor & Target molecule & Clinical trial & References \\
\hline Glycolysis & BAY-876 & GLUT1 & Preclinical & (68) \\
\hline Glycolysis & Apple polyphenol phoretin (Ph) & GLUT2 & Preclinical & (69) \\
\hline Glycolysis & Gen-27 & Hexokinase ॥ & Preclinical & $(70)$ \\
\hline Glycolysis & Silibinin & GLUT1 & in vitro & $(71)$ \\
\hline Glycolysis & Butyrate & PKM2 & Preclinical & (72) \\
\hline TCA & Butyrate & SIRT3 & Preclinical & (73) \\
\hline Glutaminolysis & I- $\gamma$-glutamyl-p-nitroanilide (GPNA) & ASCT2/SLC1A5 & Preclinical & (27) \\
\hline Glycolysis & Oxamate & LDH-A, aspartate aminotransferase & Preclinical & (74) \\
\hline Glycolysis & Galloflavin & LDH-A & Preclinical & (75) \\
\hline Glycolysis & Lonidamide & Hexokinase II & Phase ॥ & (76) \\
\hline Lipid synthesis & Orlistat & Fatty acid synthase & Preclinical & (77) \\
\hline
\end{tabular}


permeability, poor solubility, lack of selectivity, among others, precluding the use as a systemic drug (86).

The effect of different drugs with the ability to block tumor metabolism has been studied in breast cancer, such as the combination of metformin and oxamate, mTOR, and LDH-A inhibition, leading to apoptosis and autophagy activation (87). This drug combination dramatically reduced the triple-negative breast tumor growth in mice in a short time, and effectiveness lasted for 5 months after finishing the treatment. These drugs seem to act directly on tumor cells by inhibiting glycolysis and mTOR signaling and activating mechanisms that eventually drive to apoptosis. So, a metabolic shift in breast cancer affects the epigenome directly and has repercussions on gene expression and tumor development. The unraveling of these epigenetic enzymes modulated by the metabolism could serve as pharmacological targets, having a deep impact on the treatment of breast cancer.

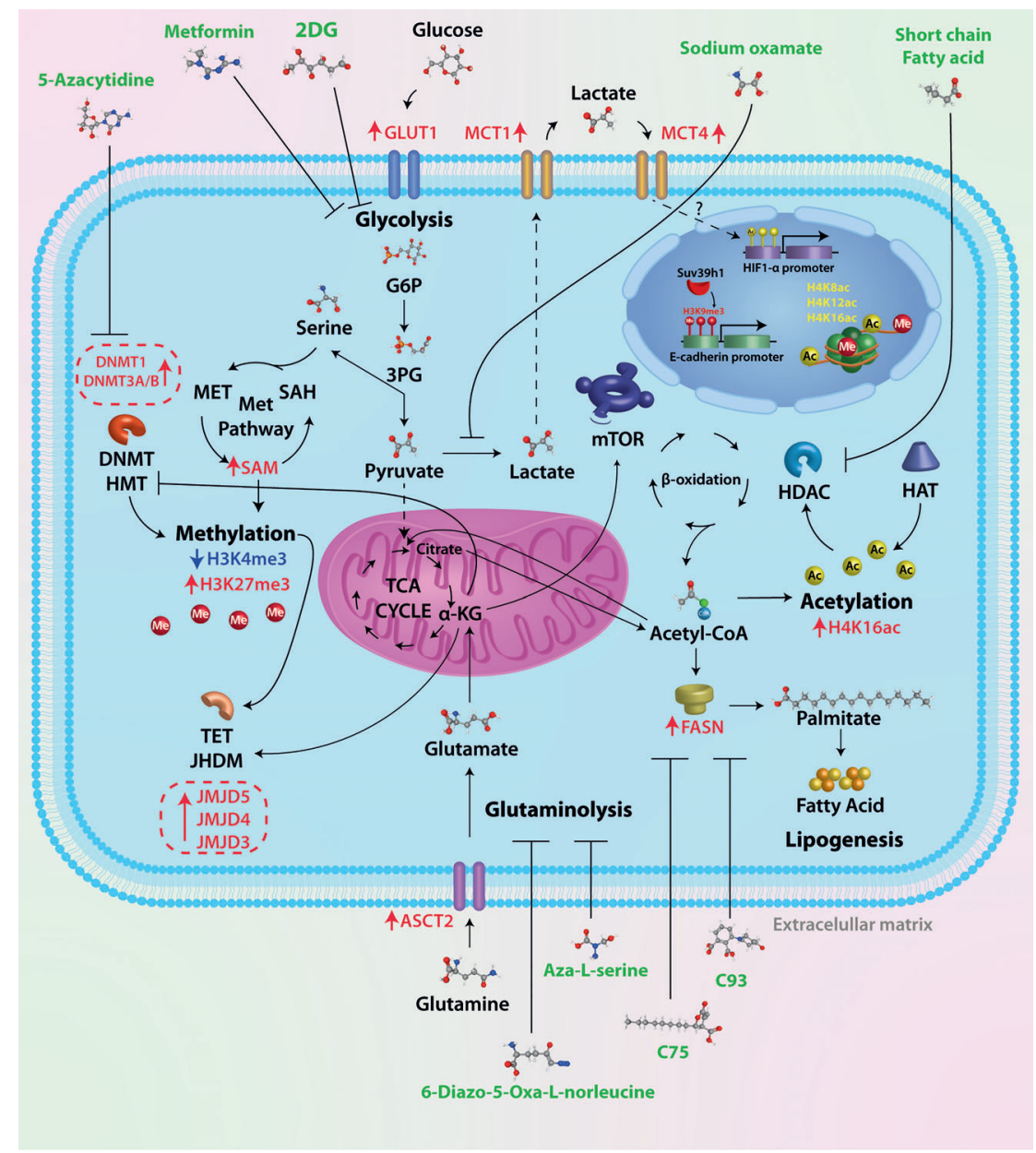

FIGURE 1 | Therapeutic targets of tumor metabolism. Tumor cells have a high glycolytic rate and overexpress key enzymes of glucose metabolism. Several drugs have been used in preclinical trials to test their effectiveness, such as 2DG, a glucose analog whose metabolic product (2-DG-P) is unable to be metabolized, therefore inhibiting glycolysis. Oxamic acid is a competitive inhibitor of LDHA, an enzyme overexpressed in breast cancer cells; the result of this inhibition is lower levels of lactate, a critical oncometabolite for cell migration and tumor progression. In tumor cells, approximately $90 \%$ of glucose is metabolized to lactate; to replenish the deficit of carbon molecules, there is an increase in glutamine metabolism. The ASCT2 transporter is overexpressed in breast cancer; glutamine is metabolized in the cytosol to glutamate and subsequently transported to the mitochondrial matrix and incorporated into TCA in the form of $\alpha$-KG. The antitumor effect of two glutamine analogs (6-diazo-5-oxa-L-norleucine and Aza-L-serine) has been shown. Acetyl-CoA is a central molecule in metabolism as it participates in catabolic (glycolysis and beta-oxidation) and anabolic reactions (lipogenesis, steroid synthesis, acetylcholine synthesis, etc.). In addition to this crucial role in cellular metabolism, Acetyl-CoA is the sole donor of acetyl groups for the acetylation of proteins and particularly histones. Histone acetylation is catalyzed by histone acetyltransferases (HATs), whereas removal of the acetyl group is mediated by histone deacetylases (HDACs). It has been shown that short-chain fatty acids such as valproic acid and butyrate, among others, can inhibit HDACs. Increased levels of acetyl-CoA promote fatty acid synthesis associated with FASN overexpression. Different inhibitors of the key enzyme in fatty acid synthesis have been used (C75, C93, and orlistat, among others), which are inhibitors of the thioesterase domain of fatty acid synthase (FASN). The epigenetic mechanism classically described is DNA methylation. The pathway that supplies methyl groups for both DNA methylation and histone and protein methylation is the one-carbon (1C) pathway metabolism, in which two distinct pathways, the folate and methionine cycle, converge, resulting in the product S-adenosylmethionine. The epigenetic mechanism classically described is DNA methylation. While no inhibitors of these metabolic pathways have been identified, several molecules have been used to inhibit the activity of enzymes involved in DNA methylation (DNMT1, DNMT3/B). 
For a summarized graphical representation of the ideas outlined in this mini-review, see Figure 1.

\section{AUTHOR CONTRIBUTIONS}

Conceptualization: JC-H, EP-Y, CP-P. Writing-Original Draft: CP-P, JC-H, EP-Y, and NJ-H. Preparation: ID-W, CC-R, and DC-L. Writing-Review and Editing: CP-P and NJ-H. All

\section{REFERENCES}

1. Zheng J. Energy Metabolism of Cancer: Glycolysis Versus Oxidative Phosphorylation (Review). Oncol Lett (2012) 4:1151-7. doi: 10.3892/ ol.2012.928

2. Spencer NY, Stanton RC. The Warburg Effect, Lactate, and Nearly a Century of Trying to Cure Cancer. Semin Nephrol (2019) 39:380-93. doi: 10.1016/ j.semnephrol.2019.04.007

3. Valvona CJ, Fillmore HL, Nunn PB, Pilkington GJ. The Regulation and Function of Lactate Dehydrogenase A: Therapeutic Potential in Brain Tumor. Brain Pathol (2016) 26:3-17. doi: 10.1111/bpa.12299

4. Sun L, Suo C, ting L, Zhang H, Gao P. Metabolic Reprogramming for Cancer Cells and Their Microenvironment: Beyond the Warburg Effect. Biochim Biophys Acta Rev Cancer (2018) 1870:51-66. doi: 10.1016/j.bbcan.2018.06.005

5. Ward PS, Thompson CB. Signaling in Control of Cell Growth and Metabolism. Cold Spring Harb Perspect Biol (2012) 4:1-15. doi: 10.1101/ cshperspect.a006783

6. Thakur C, Chen F. Connections Between Metabolism and Epigenetics in Cancers. Semin Cancer Biol (2019) 57:52-8. doi: 10.1016/j.semcancer. 2019.06.006

7. Martinez-Outschoorn UE, Peiris-Pagés M, Pestell RG, Sotgia F, Lisanti MP. Cancer Metabolism: A Therapeutic Perspective. Nat Rev Clin Oncol (2017) 14:11-31. doi: 10.1038/nrclinonc.2016.60

8. Deng Y, Zou J, Deng T, Liu J. Clinicopathological and Prognostic Significance of GLUT1 in Breast Cancer A Meta-Analysis. Medicine (United States) (2018) 97:1-6. doi: 10.1097/MD.0000000000012961

9. Cairns RA, Harris IS, Mak TW. Regulation of Cancer Cell Metabolism. Nat Rev Cancer (2011) 11:85-95. doi: 10.1038/nrc2981

10. Liu GM, Zhang YM. Targeting FBPase Is an Emerging Novel Approach for Cancer Therapy. Cancer Cell Int (2018) 18:1-16. doi: 10.1186/s12935-0180533-z

11. Latham T, MacKay L, Sproul D, Karim M, Culley J, Harrison DJ, et al. Lactate, a Product of Glycolytic Metabolism, Inhibits Histone Deacetylase Activity and Promotes Changes in Gene Expression. Nucleic Acids Res (2012) 40:4794-803. doi: 10.1093/nar/gks066

12. Willkomm L, Gehlert S, Jacko D, Schiffer T, Bloch W. P38 MAPK Activation and H3K4 Trimethy Lation is Decreased by Lactate In Vitro and High Intensity Resistance Training in Human Skeletal Muscle. PLoS One (2017) 12:1-16. doi: 10.1371/journal.pone.0176609

13. Becker LM, O'Connell JT, Vo AP, Cain MP, Tampe D, Bizarro L, et al. Epigenetic Reprogramming of Cancer-Associated Fibroblasts Deregulates Glucose Metabolism and Facilitates Progression of Breast Cancer. Cell Rep (2020) 31:1-24. doi: 10.1016/j.celrep.2020.107701

14. Anderson NM, Mucka P, Kern JG, Feng H. The Emerging Role and Targetability of the TCA Cycle in Cancer Metabolism. Protein Cell (2018) 9:216-37. doi: 10.1007/s13238-017-0451-1

15. Islam RA, Hossain S, Chowdhury EH. Potential Therapeutic Targets in Energy Metabolism Pathways of Breast Cancer. Curr Cancer Drug Targets (2017) 17:1-12. doi: 10.2174/1568009617666170330150458

16. Pietrocola F, Galluzzi L, Bravo-San Pedro JM, Madeo F, Kroemer G. Acetyl Coenzyme A: A Central Metabolite and Second Messenger. Cell Metab (2015) 21:805-21. doi: 10.1016/j.cmet.2015.05.014

17. Ropero S, Esteller M. The Role of Histone Deacetylases (HDACs) in Human Cancer. Mol Oncol (2007) 1:19-25. doi: 10.1016/j.molonc.2007.01.001 authors contributed to the article and approved the submitted version.

\section{ACKNOWLEDGMENTS}

CC- $\mathrm{R}$ is a student of Programa de Doctorado en Ciencias Biologicas, belonging to Universidad Nacional Autónoma de México.

18. Messier TL, Gordon JAR, Boyd JR, Tye CE, Browne G, Stein JL, et al. Histone H3 Lysine 4 Acetylation and Methylation Dynamics Define Breast Cancer Subtypes. Oncotarget (2016) 7:5094-109. doi: 10.18632/oncotarget.6922

19. Chen Y, Qian J, He Q, Zhao H, Toral-Barza L, Shi C, et al. mTOR Complex-2 Stimulates Acetyl-CoA and De Novo Lipogenesis Through ATP Citrate Lyase in HER2/PIK3CA-Hyperactive Breast Cancer. Oncotarget (2016) 7:25224-40. doi: 10.18632/oncotarget.8279

20. Corominas-Faja B, Cuyàs E, Gumuzio J, Bosch-Barrera J, Leis O, Martin ÁG, et al. Chemical Inhibition of Acetyl-CoA Carboxylase Suppresses SelfRenewal Growth of Cancer Stem Cells. Oncotarget (2014) 5:8306-16. doi: 10.18632/oncotarget.2059

21. Menzies KJ, Zhang H, Katsyuba E, Auwerx J. Protein Acetylation in Metabolism-Metabolites and Cofactors. Nat Rev Endocrinol (2016) 12:4360. doi: $10.1038 /$ nrendo.2015.181

22. Wan J, Zhan J, Li S, Ma J, Xu W, Liu C, et al. PCAF-Primed EZH2 Acetylation Regulates its Stability and Promotes Lung Adenocarcinoma Progression. Nucleic Acids Res (2015) 43:3591-604. doi: 10.1093/nar/gkv238

23. Wang HJ, Hsieh YJ, Cheng WC, Lin CP, Lin YS, Yang SF, et al. JMJD5 Regulates PKM2 Nuclear Translocation and Reprograms HIF-1 $\alpha$-Mediated Glucose Metabolism. Proc Natl Acad Sci U S A (2014) 111:279-84. doi: $10.1073 /$ pnas.1311249111

24. Garcia J, Lizcano F. KDM4C Activity Modulates Cell Proliferation and Chromosome Segregation in Triple-Negative Breast Cancer. Breast Cancer Basic Clin Res (2016) 10:169-75. doi: 10.4137/BCBCR.S40182

25. Yang L, Venneti S, Nagrath D. Glutaminolysis: A Hallmark of Cancer Metabolism. Annu Rev BioMed Eng (2017) 19:163-94. doi: 10.1146/ annurev-bioeng-071516-044546

26. Liu Y, Zhao T, Li Z, Wang L, Yuan S, Sun L. The Role of ASCT2 in Cancer: A Review. Eur J Pharmacol (2018) 837:81-7. doi: 10.1016/j.ejphar.2018.07.007

27. Van Geldermalsen M, Wang Q, Nagarajah R, Marshall AD, Thoeng A, Gao D, et al. ASCT2/SLC1A5 Controls Glutamine Uptake and Tumour Growth in Triple-Negative Basal-Like Breast Cancer. Oncogene (2016) 35:3201-8. doi: 10.1038/onc.2015.381

28. Zhang Z, Liu R, Shuai Y, Huang Y, Jin R, Wang X, et al. ASCT2 (SLC1A5)Dependent Glutamine Uptake Is Involved in the Progression of Head and Neck Squamous Cell Carcinoma. Br J Cancer (2020) 122:82-93. doi: 10.1038/ s41416-019-0637-9

29. Masisi BK, El Ansari R, Alfarsi L, Rakha EA, Green AR, Craze ML. The Role of Glutaminase in Cancer. Histopathology (2020) 76:498-508. doi: 10.1111/ his. 14014

30. Cassago A, Ferreira APS, Ferreira IM, Fornezari C, Gomes ERM, Greene KS, et al. Mitochondrial Localization and Structure-Based Phosphate Activation Mechanism of Glutaminase C With Implications for Cancer Metabolism. Proc Natl Acad Sci U S A (2012) 109:1092-7. doi: 10.1073/pnas.1112495109

31. Szeliga M, Bogacińska-Karaś M, Różycka A, Hilgier W, Marquez J, Albrecht J. Silencing of GLS and Overexpression of GLS2 Genes Cooperate in Decreasing the Proliferation and Viability of Glioblastoma Cells. Tumor Biol (2014) 35:1855-62. doi: 10.1007/s13277-013-1247-4

32. Van Den Heuvel APJ, Jing J, Wooster RF, Bachman KE. Analysis of Glutamine Dependency in non-Small Cell Lung Cancer: GLS1 Splice Variant GAC Is Essential for Cancer Cell Growth. Cancer Biol Ther (2012) 13:1185-94. doi: 10.4161/cbt.21348

33. Yu D, Shi X, Meng G, Chen J, Yan C, Jiang Y, et al. Kidney-Type Glutaminase (GLS1) Is a Biomarker for Pathologic Diagnosis and Prognosis of 
Hepatocellular Carcinoma. Oncotarget (2015) 6:7619-31. doi: 10.18632/ oncotarget.3196

34. Zhang J, Mao S, Guo Y, Wu Y, Yao X, Huang Y. Inhibition of GLS Suppresses Proliferation and Promotes Apoptosis in Prostate Cancer. Biosci Rep (2019) 39:1-10. doi: 10.1042/BSR20181826

35. Timmerman LA, Holton T, Yuneva M, Louie RJ, Padró M, Daemen A, et al. Glutamine Sensitivity Analysis Identifies the xCT Antiporter as a Common Triple-Negative Breast Tumor Therapeutic Target. Cancer Cell (2013) 24:45065. doi: 10.1016/j.ccr.2013.08.020

36. Craze ML, Cheung H, Jewa N, Coimbra NDM, Soria D, El-Ansari R, et al. MYC Regulation of Glutamine-Proline Regulatory Axis Is Key in Luminal B Breast Cancer. Br J Cancer (2018) 118:258-65. doi: 10.1038/bjc.2017.387

37. Lampa M, Arlt H, He T, Ospina B, Reeves J, Zhang B, et al. Glutaminase is Essential for the Growth of Triple-Negative Breast Cancer Cells With a Deregulated Glutamine Metabolism Pathway and Its Suppression Synergizes With mTOR Inhibition. PLoS One (2017) 12:e0185092. doi: 10.1371/journal.pone.0185092

38. Sellers K, Fox MP, Ii MB, Slone SP, Higashi RM, Miller DM, et al. Pyruvate Carboxylase Is Critical for Non-Small-Cell Lung Cancer Proliferation. J Clin Invest (2015) 125:687-98. doi: 10.1172/JCI72873

39. Altman BJ, Stine ZE, Dang CV. From Krebs to Clinic: Glutamine Metabolism to Cancer Therapy. Nat Rev Cancer (2016) 16:619-34. doi: 10.1038/ nrc.2016.71

40. Durán RV, Oppliger W, Robitaille AM, Heiserich L, Skendaj R, Gottlieb E, et al. Glutaminolysis Activates Rag-Mtorc1 Signaling. Mol Cell (2012) 47:34958. doi: 10.1016/j.molcel.2012.05.043

41. Yang L, Moss T, Mangala LS, Marini J, Zhao H, Wahlig S, et al. Metabolic Shifts Toward Glutamine Regulate Tumor Growth, Invasion and Bioenergetics in Ovarian Cancer. Mol Syst Biol (2014) 10:81-7. doi: 10.1002/msb.20134892

42. Liu G, Zhu J, Yu M, Cai C, Zhou Y, Yu M, et al. Glutamate Dehydrogenase is a Novel Prognostic Marker and Predicts Metastases in Colorectal Cancer Patients. J Transl Med (2015) 13:1-10. doi: 10.1186/s12967-015-0500-6

43. Dang L, White DW, Gross S, Bennett BD, Bittinger MA, Driggers EM, et al. Cancer-Associated IDH1 Mutations Produce 2-Hydroxyglutarate. Nature (2009) 462:739-44. doi: 10.1038/nature08617

44. Seltzer MJ, Bennett BD, Joshi AD, Gao P, Thomas AG, Ferraris DV, et al. Inhibition of Glutaminase Preferentially Slows Growth of Glioma Cells With Mutant IDH1. Cancer Res (2010) 70:8981-7. doi: 10.1158/0008-5472.CAN10-1666

45. Bai L, Bernard K, Tang X, Hu M, Horowitz JC, Thannickal VJ, et al. Glutaminolysis Epigenetically Regulates Antiapoptotic Gene Expression in Idiopathic Pulmonary Fibrosis Fibroblasts. Am J Respir Cell Mol Biol (2019) 60:49-57. doi: 10.1165/rcmb.2018-01800C

46. Katt WP, Ramachandran S, Erickson JW, Cerione RA. Dibenzophenanthridines as Inhibitors of Glutaminase C and Cancer Cell Proliferation. Mol Cancer Ther (2012) 11:1269-78. doi: 10.1158/15357163.MCT-11-0942

47. Simpson NE, Tryndyak VP, Beland FA, Pogribny IP. An In Vitro Investigation of Metabolically Sensitive Biomarkers in Breast Cancer Progression. Breast Cancer Res Treat (2012) 133:959-68. doi: 10.1007/ S10549-011-1871-X

48. Jin L, Alesi GN, Kang S. Glutaminolysis as a Target for Cancer Therapy. Oncogene (2016) 35:3619-25. doi: 10.1038/onc.2015.447

49. Gruslova A, McClellan B, Balinda HU, Viswanadhapalli S, Alers V, Sareddy GR, et al. FASN Inhibition as a Potential Treatment for Endocrine-Resistant Breast Cancer. Breast Cancer Res Treat (2021) 187:375-86. doi: 10.1007/ s10549-021-06231-6

50. Menendez JA, Papadimitropoulou A, Vander ST, Cuyàs E, Oza-Gajera BP, Verdura S, et al. Fatty Acid Synthase Confers Tamoxifen Resistance to Er+/Her2+ Breast Cancer. Cancers (Basel) (2021) 13:1-19. doi: 10.3390/ cancers 13051132

51. Ferraro GB, Ali A, Luengo A, Kodack DP, Deik A, Abbott KL, et al. Fatty Acid Synthesis Is Required for Breast Cancer Brain Metastasis. Nat Cancer (2021) 2:414-28. doi: 10.1038/s43018-021-00183-y

52. Jin Q, Yuan LX, Boulbes D, Baek JM, Wang YN, Gomez-Cabello D, et al. Fatty Acid Synthase Phosphorylation: A Novel Therapeutic Target in HER2Overexpressing Breast Cancer Cells. Breast Cancer Res (2010) 12:216-37. doi: $10.1186 /$ bcr 2777
53. Tumanov S, Bulusu V, Kamphorst JJ. Analysis of Fatty Acid Metabolism Using Stable Isotope Tracers and Mass Spectrometry. In: Methods in Enzymology (Methods Enzymol). Academic Press, Elservier (2015). p. 197217. doi: 10.1016/bs.mie.2015.05.017

54. Yang D, Peng M, Hou Y, Qin Y, Wan X, Zhu P, et al. Oxidized ATM Promotes Breast Cancer Stem Cell Enrichment Through Energy Metabolism Reprogram-Mediated Acetyl-CoA Accumulation. Cell Death Dis (2020) 11:1-17. doi: 10.1038/s41419-020-2714-7

55. Esteller M. CpG Island Hypermethylation and Tumor Suppressor Genes: A Booming Present, a Brighter Future. Oncogene (2002) 21:5427-40. doi: $10.1038 /$ sj.onc. 1205600

56. Bogdanović O, Veenstra GJC. DNA Methylation and Methyl-CpG Binding Proteins: Developmental Requirements and Function. Chromosoma (2009) 118:549-65. doi: 10.1007/s00412-009-0221-9

57. Bernstein BE, Mikkelsen TS, Xie X, Kamal M, Huebert DJ, Cuff J, et al. A Bivalent Chromatin Structure Marks Key Developmental Genes in Embryonic Stem Cells. Cell (2006) 125:315-26. doi: 10.1016/j.cell.2006.02.041

58. Shyh-Chang N, Locasale JW, Lyssiotis CA, Zheng Y, Teo RY, Ratanasirintrawoot S, et al. Influence of Threonine Metabolism on SAdenosylmethionine and Histone Methylation. Science (80) (2013) 339:222-6. doi: 10.1126/science.1226603

59. Robertson KD, Uzvolgyi E, Liang G, Talmadge C, Sumegi J, Gonzales FA, et al. The Human DNA Methyltransferases (DNMTs) 1, 3a and 3b: Coordinate mRNA Expression in Normal Tissues and Overexpression in Tumors. Nucleic Acids Res (1999) 27:2291-8. doi: 10.1093/nar/27.11.2291

60. Girault I, Tozlu S, Lidereau R, Bièche I. Expression Analysis of DNA Methyltransferases 1, 3A, and 3B in Sporadic Breast Carcinomas. Clin Cancer Res (2003) 9:4415-22.

61. Paydar P, Asadikaram G, Nejad HZ, Akbari H, Abolhassani M, Moazed V, et al. Epigenetic Modulation of BRCA-1 and MGMT Genes, and Histones H4 and H3 are Associated With Breast Tumors. J Cell Biochem (2019) 120:1372636. doi: $10.1002 /$ jcb. 28645

62. Hyun K, Jeon J, Park K, Kim J. Writing, Erasing and Reading Histone Lysine Methylations. Exp Mol Med (2017) 49:1-17. doi: 10.1038/emm.2017.11

63. Michalak EM, Visvader JE. Dysregulation of Histone Methyltransferases in Breast Cancer - Opportunities for New Targeted Therapies? Mol Oncol (2016) 10:1497. doi: 10.1016/j.molonc.2016.09.003

64. Borrego SL, Fahrmann J, Datta R, Stringari C, Grapov D, Zeller M, et al. Metabolic Changes Associated With Methionine Stress Sensitivity in MDA-MB-468 Breast Cancer Cells. Cancer Metab (2016) 4. doi: 10.1186/s40170-016-0148-6

65. Dong C, Wu Y, Wang Y, Wang C, Kang T, Rychahou PG, et al. Interaction With Suv39H1 is Critical for Snail-Mediated E-Cadherin Repression in Breast Cancer. Oncogene (2013) 32:1351-62. doi: 10.1038/onc.2012.169

66. Macaluso M, Cinti C, Russo G, Russo A, Giordano A. Prb2/P130-E2F4/5HDAC1-SUV39H1-P300 and Prb2/P130-E2F4/5-HDAC1-SUV39H1DNMT1 Multimolecular Complexes Mediate the Transcription of Estrogen Receptor- $\alpha$ in Breast Cancer. Oncogene (2003) 22:3511-7. doi: 10.1038/ sj.onc. 1206578

67. Pavlova NN, Thompson CB. The Emerging Hallmarks of Cancer Metabolism. Cell Metab (2016) 23:27-47. doi: 10.1016/j.cmet.2015.12.006

68. Wu Q, Heidenreich D, Zhou S, Ackloo S, Krämer A, Nakka K, et al. A Chemical Toolbox for the Study of Bromodomains and Epigenetic Signaling. Nat Commun (2019) 10:1-14. doi: 10.1038/s41467-019-09672-2

69. Wu KH, Ho CT, Chen ZF, Chen LC, Whang-Peng J, Lin TN, et al. The Apple Polyphenol Phloretin Inhibits Breast Cancer Cell Migration and Proliferation via Inhibition of Signals by Type 2 Glucose Transporter. J Food Drug Anal (2018) 26:221-31. doi: 10.1016/j.jfda.2017.03.009

70. Tao L, Liu Y, Ding Y, Liu X, Zhang X, Hu R, et al. Gen-27, a Newly Synthesized Flavonoid, Inhibits Glycolysis and Induces Cell Apoptosis via Suppression of Hexokinase II in Human Breast Cancer Cells. Biochem Pharmacol (2017) 125:12-25. doi: 10.1016/j.bcp.2016.11.001

71. Deep G, Agarwal R. Targeting Tumor Microenvironment With Silibinin: Promise and Potential for a Translational Cancer Chemopreventive Strategy. Curr Cancer Drug Targets (2013) 13:486-99. doi: 10.2174/15680096113139990041

72. Li Q, Cao L, Tian Y, Zhang P, Ding C, Lu W, et al. Butyrate Suppresses the Proliferation of Colorectal Cancer Cells via Targeting Pyruvate Kinase M2 and Metabolic Reprogramming. Mol Cell Proteomics (2018) 17:1531-45. doi: $10.1074 /$ mcp.RA118.000752 
73. Xu S, Liu CX, Xu W, Huang L, Zhao JY, Zhao SM. Butyrate Induces Apoptosis by Activating PDC and Inhibiting Complex I Through SIRT3 Inactivation. Signal Transduct Target Ther (2017) 2:1-9. doi: 10.1038/sigtrans.2016.35

74. Thornburg JM, Nelson KK, Clem BF, Lane AN, Arumugam S, Simmons A, et al. Targeting Aspartate Aminotransferase in Breast Cancer. Breast Cancer Res (2008) 10:1-12. doi: 10.1186/bcr2154

75. Farabegoli F, Vettraino M, Manerba M, Fiume L, Roberti M, Di Stefano G. Galloflavin, a New Lactate Dehydrogenase Inhibitor, Induces the Death of Human Breast Cancer Cells With Different Glycolytic Attitude by Affecting Distinct Signaling Pathways. Eur J Pharm Sci (2012) 47:729-38. doi: 10.1016/ j.ejps.2012.08.012

76. Nath K, Guo L, Nancolas B, Nelson DS, Shestov AA, Lee SC, et al. Mechanism of Antineoplastic Activity of Lonidamine. Biochim Biophys Acta Rev Cancer (2016) 1866:151-62. doi: 10.1016/j.bbcan.2016.08.001

77. Menendez JA, Vellon L, Lupu R. Antitumoral Actions of the Anti-Obesity Drug Orlistat $\left(\right.$ Xenical $^{\mathrm{TM}}$ ) in Breast Cancer Cells: Blockade of Cell Cycle Progression, Promotion of Apoptotic Cell Death and PEA3-Mediated Transcriptional Repression of Her2/neu (erbB-2) Oncogene. Ann Oncol (2005) 16:1253-67. doi: 10.1093/annonc/mdi239

78. Al-Shammari AM, Abdullah AH, Allami ZM, Yaseen NY. 2-Deoxyglucose and Newcastle Disease Virus Synergize to Kill Breast Cancer Cells by Inhibition of Glycolysis Pathway Through Glyceraldehyde3-Phosphate Downregulation. Front Mol Biosci (2019) 6:90. doi: 10.3389/fmolb.2019.00090

79. Cheng G, Zielonka J, Dranka BP, McAllister D, Mackinnon AC, Joseph J, et al. Mitochondria-Targeted Drugs Synergize With 2-Deoxyglucose to Trigger Breast Cancer Cell Death. Cancer Res (2012) 72:2634-44. doi: 10.1158/ 0008-5472.CAN-11-3928

80. Aghaee F, Islamian JP, Baradaran B. Enhanced Radiosensitivity and Chemosensitivity of Breast Cancer Cells by 2-Deoxy-D-Glucose in Combination Therapy. J Breast Cancer (2012) 15:141-7. doi: 10.4048/jbc.2012.15.2.141

81. Wang JB, Erickson JW, Fuji R, Ramachandran S, Gao P, Dinavahi R, et al. Targeting Mitochondrial Glutaminase Activity Inhibits Oncogenic Transformation. Cancer Cell (2010) 18:207-19. doi: 10.1016/j.ccr.2010.08.009

82. Shukla K, Ferraris DV, Thomas AG, Stathis M, Duvall B, Delahanty G, et al. Design, Synthesis, and Pharmacological Evaluation of Bis-2-(5Phenylacetamido-1,2,4-Thiadiazol-2-Yl)Ethyl Sulfide 3 (BPTES) Analogs as
Glutaminase Inhibitors. J Med Chem (2012) 55:10551-63. doi: 10.1021/ jm301191p

83. Gross MI, Demo SD, Dennison JB, Chen L, Chernov-Rogan T, Goyal B, et al. Antitumor Activity of the Glutaminase Inhibitor CB-839 in Triple-Negative Breast Cancer. Mol Cancer Ther (2014) 13:890-901. doi: 10.1158/1535-7163.MCT-13-0870

84. Puig T, Turrado C, Benhamú B, Aguilar H, Relat J, Ortega-Gutiérrez S, et al. Novel Inhibitors of Fatty Acid Synthase With Anticancer Activity. Clin Cancer Res (2009) 15:7608-15. doi: 10.1158/1078-0432.CCR-09-0856

85. Pizer ES, Wood FD, Heine HS, Romantsev FE, Pasternack GR, Kuhajda FP, et al. Inhibition of Fatty Acid Synthesis Delays Disease Progression in a Xenograft Model of Ovarian Cancer. Cancer Res (1996) 56:1189-93.

86. Flavin R, Peluso S, Nguyen PL, Loda M. Fatty Acid Synthase as a Potential Therapeutic Target in Cancer. Futur Oncol (2010) 6:551-62. doi: 10.2217/ fon. 10.11

87. García-Castillo V, López-Urrutia E, Villanueva-Sánchez O, Ávila-Rodríguez MA, Zentella-Dehesa A, Cortés-González C, et al. Targeting Metabolic Remodeling in Triple Negative Breast Cancer in a Murine Model. J Cancer (2017) 8:178-89. doi: 10.7150/jca.16387

Conflict of Interest: The authors declare that the research was conducted in the absence of any commercial or financial relationships that could be construed as a potential conflict of interest.

Publisher's Note: All claims expressed in this article are solely those of the authors and do not necessarily represent those of their affiliated organizations, or those of the publisher, the editors and the reviewers. Any product that may be evaluated in this article, or claim that may be made by its manufacturer, is not guaranteed or endorsed by the publisher.

Copyright (C) 2021 Coronel-Hernández, Pérez-Yépez, Delgado-Waldo, ContrerasRomero, Jacobo-Herrera, Cantú-De León and Pérez-Plasencia. This is an open-access article distributed under the terms of the Creative Commons Attribution License (CC BY). The use, distribution or reproduction in other forums is permitted, provided the original author(s) and the copyright owner(s) are credited and that the original publication in this journal is cited, in accordance with accepted academic practice. No use, distribution or reproduction is permitted which does not comply with these terms. 